\title{
Development of In-Pipe Robot D300: Cornering Mechanism
}

\author{
Ana Sakura Zainal Abidin ${ }^{a}$, Sim Chia Chie, Muhammad Hamizan Zaini, Muhammad Farhan Aiman Mohd Pauzi, \\ Mohammad Muaz Sadini, Shahrol Mohamaddan, Annisa Jamali, Rasli Muslimen, Muhd Fadzli Ashari, Mohd Syahmi \\ Jamaludin
}

Department of Mechanical and Manufacturing Engineering, Universiti Malaysia Sarawak, Sarawak, Malaysia

\begin{abstract}
Utilization of robots for in-pipe maintenance considered as one of the most effective ways to improve the pipeline operation. The specific condition and structure of a pipeline system is a challenge for the robots to perform its task. This research aims to assess the characteristic and performance of the in-pipe robot in a $90^{\circ}$ pipe elbow. Hence, a prototype of in-pipe robot for $300 \mathrm{~mm}$ UPVC pipe diameter (IPR-D300) has been built. The conception of the robot is based on wall-pressed caterpillar type which is suitable for not only horizontal pipeline position, but also vertical and elbows pipeline. This robot is equipped with ultrasonic sensors, high performance microcontroller board and powerful geared motor for travelling in the corner of pipeline. The developed IPR-D300 consist of three independent driving modules that are able to alter the speed according to the pipeline characteristic. The hardware and software of the IPR-D300 performance have been tested and successfully perform the cornering task smoothly. The developed IPR-D300 potentially can be applied for in-pipe cleaning operation especially on centralised sewerage system. As standard pipeline size for sewerage system housing in Malaysia is $200 \mathrm{~mm}$, smaller and robust in-pipe robot will be the future target.
\end{abstract}

\section{Introduction}

Pipeline system has been employed as the main utilities in most nations for an extensive period of time [1]. This is because shipping with pipeline system has a lower cost per unit and higher capacity which is the most economical way to transport large quantities of flowing products. However, many troubles occurred in pipelines for the long-term erosion of transport materials and environmental effects namely blockage, crack, corrosion and mechanical damage [2]. Thus, regular cleaning and inspection are needed to ensure the safety, integrity and maximum efficiency of the operation of pipeline systems. Nevertheless, it becomes a challenging issue as it is tough to detect the area of crack or clog inside the pipes that are buried underground. Besides that, the maintenance and service to fix the damage manually by labours is very time consuming, less effective and costly.

Currently, the utilization of robots to do the maintenance of the pipeline is considered as one of the most effective ways to solve the problems [3]. Basically, a robot is able to move freely in a pipeline that enable it to perform more accurate checking and perform maintenance task within short period of time with lower operation cost. However, specific condition and structure of a pipeline system had caused the robots to hardly achieve high applicability. Lots of research about the locomotion of in-pipe robot have been studied to solve different condition and structure of pipeline system.
Therefore, this research has been conducted with the aim to develop an in-pipe robot that can navigate smoothly during cornering. This research limits to specific scope namely; (1) the cornering angle is $90^{\circ}$ elbow (2) the diameter of pipeline is $300 \mathrm{~mm}$ (3) material of pipeline is UPVC. This paper repots on the development of the inpipe robot and performance of the developed in-pipe robot during cornering.

In-pipe robots have been around since many years ago. Prior research have shown many mechanisms and designs have been developed by scientists and inventors from all over the world. Basically, the in-pipe robots can be classified into seven categories according to their driving mode and design namely Pig type, Wheel type, Caterpillar type, Wall-press type, Walking type, Inchworm type and Screw type [4]. There are also designed robots, Stickybot and Mini-Whegs that mimic the nature creatures (insects and lizards) that are able to climb $[5,6]$. Locomotion of the in-pipe robot is chosen base on their designated job and application. Some robot may implement two or more types of locomotion at once in order to improve its performance and efficiency in certain applications.

As compared to the wheel-based robot, caterpillar tracks is more suitable to drive on unsteady surface. This is because the caterpillar tracks is able to distribute the robot's weight evenly over a larger surface of the track where the wheel-based robot of the same potential would fail to do the job. Meanwhile, larger surface area of

\footnotetext{
a Corresponding author: zaasakura@unimas.my
} 
caterpillar tracks provides additional grip compared to the wheel-based. This feature of the caterpillar tracks is good in the situation that having steeper surfaces gradients and slippery textures [7]. Research by Jong-Hoon Kim BE [8] from Seoul National University of Technology reports that most of the wheel-based cars has the ground pressure of around $207 \mathrm{kPa}$, whereas for a seventy-ton M1 Abrams tank has the ground pressure of around $103 \mathrm{kPa}$, which is half ground pressure as compared to a car. Thus, caterpillar track helps the robot to exert less pressure in a horizontal pipeline. However, the huge friction force from the caterpillar track dramatically reduces its top speed, but this friction is seriously needed for vertical movement in pipeline. The large surface area of caterpillar track which provides lesser ground pressure is implemented to minimize risk of damage to the inner surface of the pipeline. Hence, the caterpillar-based offers more advantages as compared to wheel-based for an inpipe robot application.

\section{Methodology}

Figures 1 and 2 show conceptual design of IPR-D300 that consists of main body, three foldable links and three caterpillar wheel tracks. The IPR-D300 is developed as a wall-pressed caterpillar wheel type of in-pipe robot. Figure 2 shows an isometric view of the design. Caterpillar-based provides abundant gripping force that enable it to tackle the wall without any slipping [9]. The three foldable links improve the wall-pressed mechanism that can slightly compress or expand the caterpillar wheels to fit into the limited space especially in the elbow of pipeline. Additionally, this robot applying a tri-axial differential mechanism as the three caterpillar wheel tracks are arranged at an angle of $120^{\circ}$ with respect to one another as shown in Figure 1. The IPR-D300 is designed to conduct cleaning operation. In order to serve the operation, the cleaning device is attached to a shaft located at the center of the main body [10] as shown in Figure 2 (marked by red circle).

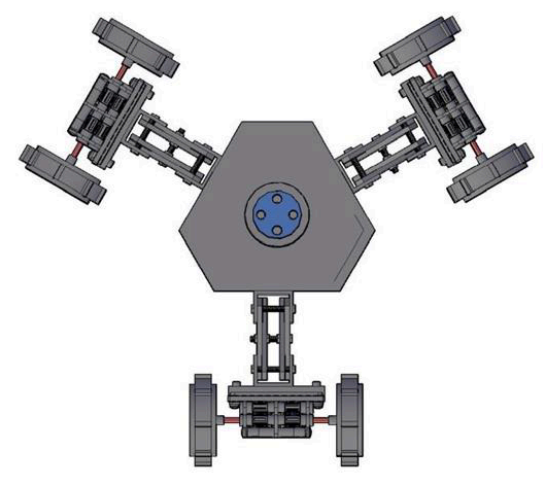

Figure 1 Conceptual design of IPR-D300, front view

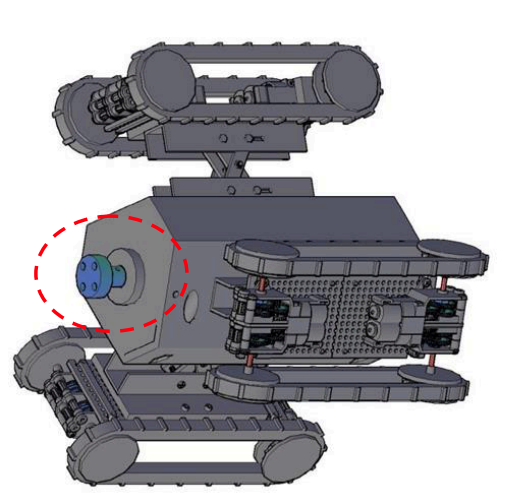

Figure 2 Conceptual design of IPR-D300, isometric view

Cornering in the pipeline is the most challenging task for any in-pipe robot to travel. Situation inside a pipeline is totally different from plain surface. A straight pipeline surface is shaped like a three-dimensionally curved surface area of cylinder, and moreover the situation becomes more complicated at the elbow as well as the branch of the pipeline. The radius of curvature $\mathrm{R}$, elbow angle $\lambda$, length of the cornering should be considered when designing the robot. The robot may get stuck or stop at the elbow if the structure of the robot is too long or too large. Therefore, it is really important to determine the correct size of a robot. The optimal length of robot inside a 12-inch pipe can be defined as [11].

$$
\mathrm{L}_{\max }=2 \sqrt{\left(R+\frac{D}{2}\right)\left(R+\frac{D}{2}\right)-\left(R+\frac{d}{2}\right)\left(R+\frac{d}{2}\right)}
$$

Where:

d, represents diameter of robot,

$\mathrm{L}_{\max }$, robot maximum length,

$\mathrm{D}$, the inner diameter of pipeline;

$\mathrm{R}$, the radius curvature of pipeline;

In this research, flexible link plays very important role in employing wall-pressed mechanism to provide additional gripping force to the wheel and to connect the main body frame and tracks as well. The main structure of flexible link is made from U shape AL-6063 bars and 3mm clear acrylic sheet (Perspex). U shaped Aluminium used as a platform while Perspex forming a cross " $\mathrm{X}$ " pattern. Figure 3 shows the conceptual design of flexible link.

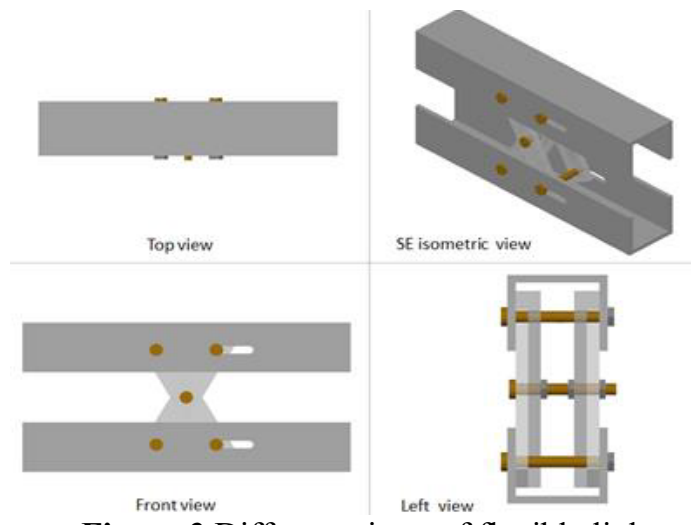

Figure 3 Different views of flexible link 
The maximum and minimum height of the link can be defined earlier by using the Pythagorean Theorem. The flexible link is developed with $12 \mathrm{~mm}$ length slots on each upper and lower U-shaped Aluminium. The designated slots enable the link to adjust its height based on the pressure applied to the wheel track. Based on the calculation, the maximum height of the flexible link can reached up to $60 \mathrm{~mm}$ at minimum slot position. During compression, the flexible link can be pushed to the maximum distance of the slot as shown in Figure 4 that consequently reduce the height of the flexible link to 50 $\mathrm{mm}$.
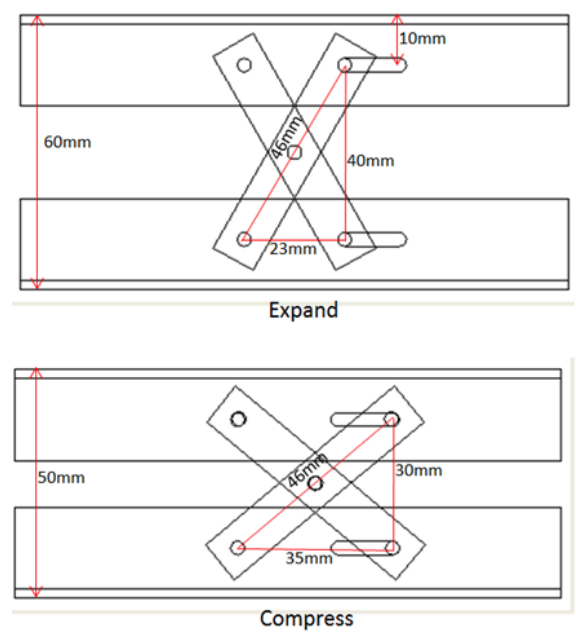

Figure 4 Adjustable dimension of flexible link

Figure 5 shows driving module of the IPR D-300 that consists of geared DC motor, wheels casing, silicone and ultrasonic sensor. In total there are four wheels powered by four geared DC motors and two silicone belts for each driving module. In order to provide higher torque to the wheel, each wheel is driven individually by a single motor and two wheels designed to drive one silicone belt. Caterpillar track is designed to have $40 \mathrm{~mm}$ wide and 180 $\mathrm{mm}$ length. This feature provides better friction grip to the wall and large surface of silicone is able to reduce damage effect to the inner wall of the pipeline. The Tamiya double gearbox is chosen as the main actuator for this driving module. The gear can be set to four different gear ratios depend on the torque needed. Thus, more options are available for the robot speed. In this case, gear ratio $344.2: 1$ with 2276 gf-cm torque is chosen to provide sufficient power to grip the inner side of the UPVC pipeline.

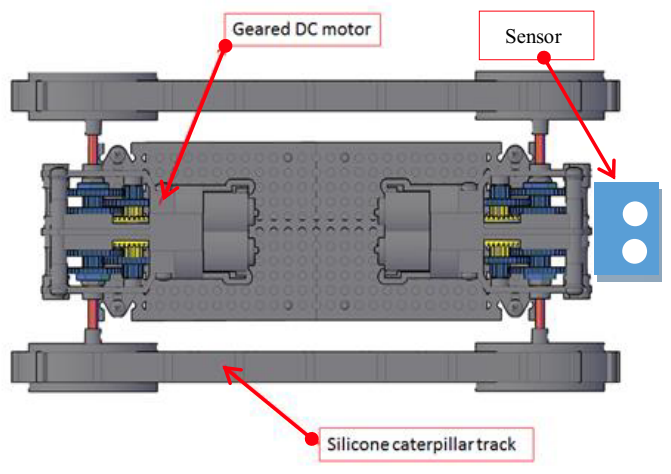

Figure 5 Parts of driving module

This section presents on the methodology of robot turning in the elbow. As mentioned previously, this robot consists of three driving module. Each module is controlled independently. By having various combinations of gear speed, the IPR-D300 is able to make a turn in the elbow. Ultrasonic sensor plays an important role in monitoring the pipeline situations, while Arduino programming will alter the speed of driving modules based on the feedback generated by the ultrasonic sensor (located at the tip of the driving module as shown in the Figure 5).

The working principle of the programming is presented in Figure 6. At the beginning of the operation in the straight pipeline, all motors run at full speed. All motors move forward at same pace until any of the sensor detects less than $6 \mathrm{~cm}$ gap from pipe wall (inner side of the cornering radius) motor of the respective driving module now shall reduce speed into half. At the same time, the other motors will run at full speed so that the IPR-D300 can create a turning effect. Logically, distance of the inner part is shorter than distance of the outer part of the elbow. Therefore, the motor of the driving module at the inner position has to reduce speed so that the other driving modules can move synchronously in the elbow. Once the IPR-D300 is out of the elbow, gap between wall and the inner driving module will be increased that consequently run the motor at full speed again. 


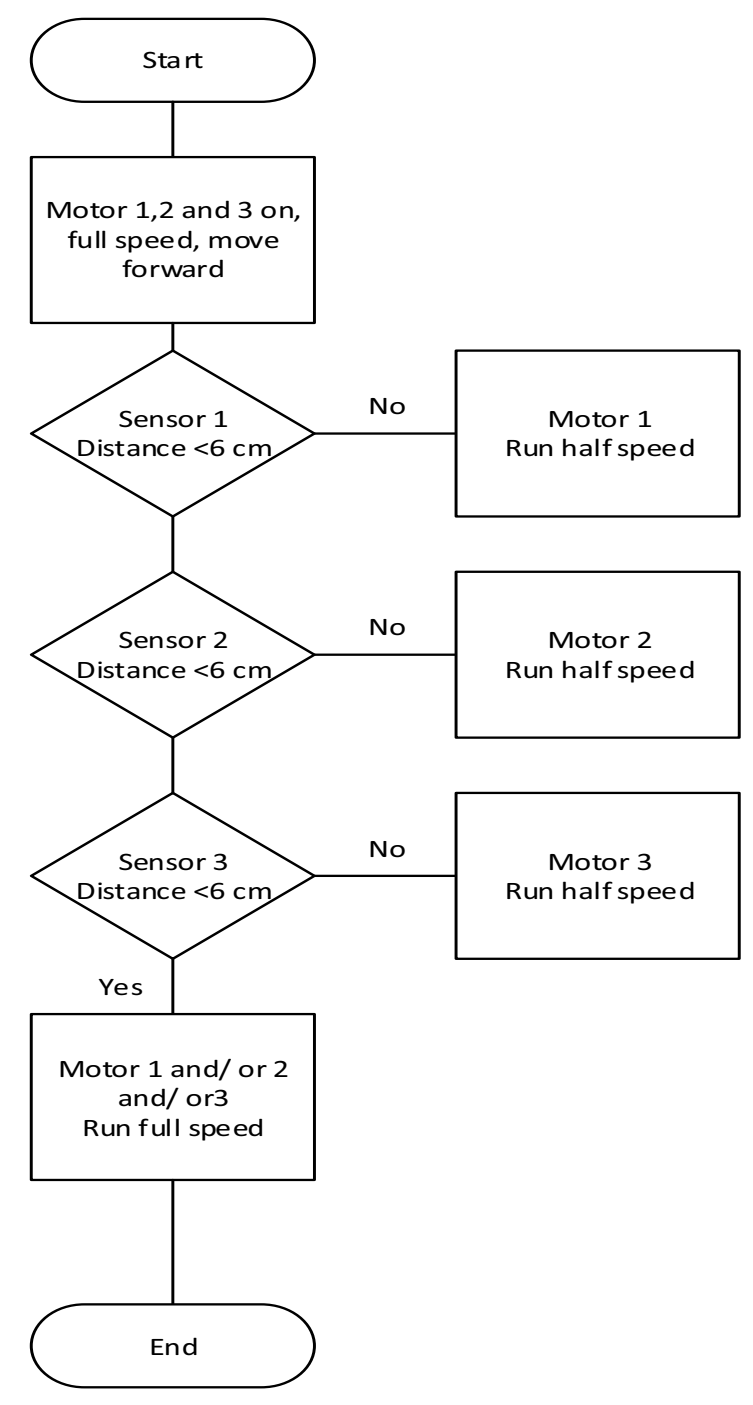

Figure 6 Flowchart for cornering operation

Figure 7 (a) until (f) show graphical simulation of the IPR-D300 during cornering in the $90^{\circ}$ elbow. The figures are arranged in sequence followed by brief explanation to elaborate the cornering operation.

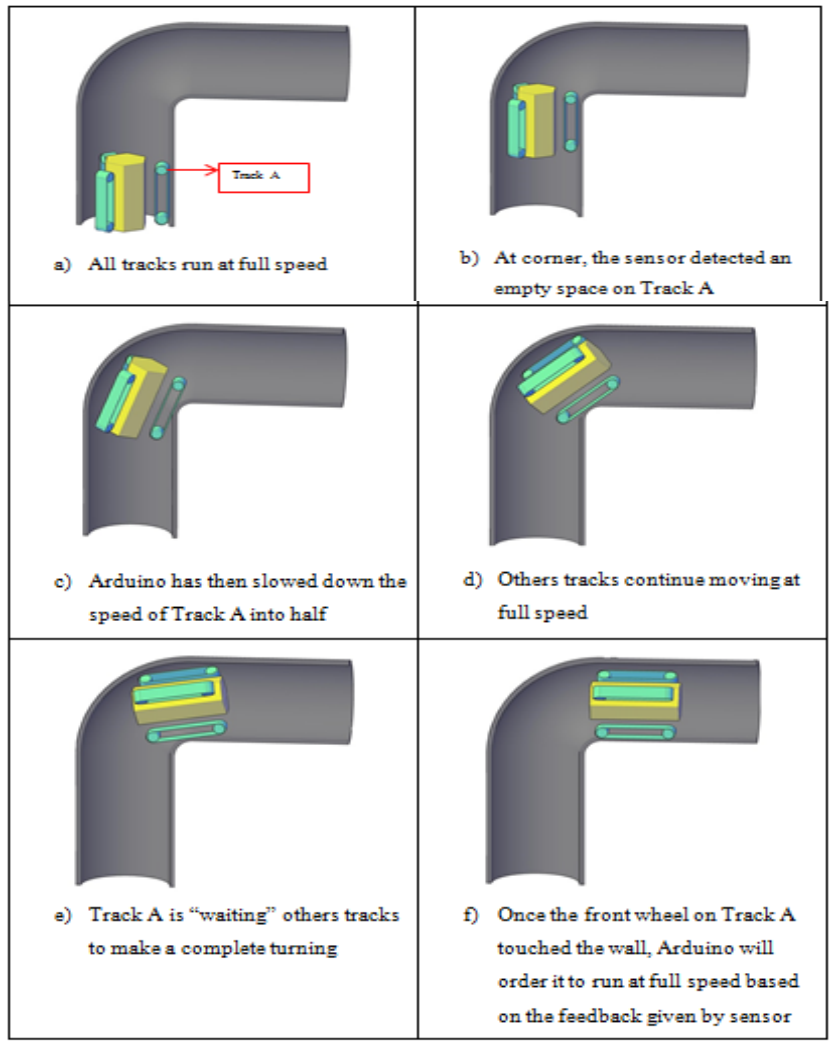

Figure 7 (a)-(f) Graphical simulation of IPR during cornering

\section{Results and discussion}

A prototype of IPR-D300 successfully assembled and perfectly fit into a 12 inch or $300 \mathrm{~mm}$ pipe diameter. Figures 8 (i) and (ii) show the experimental set up to test on the programming of the IPR-D300. Point A is a starting point located $30 \mathrm{~cm}$ from point $\mathrm{B}$. Point $\mathrm{B}$ is located at corner of the table with 1 meter height from floor. The driving module moved along the horizontal surface from point A to point B. It took 7 seconds to travel $30 \mathrm{~cm}$ distance which is equivalent to $4.29 \mathrm{~cm} / \mathrm{s}$ speed. When the module closer to point $\mathrm{B}$, the sensor detected wider gap which is beyond the designated pipeline diameter (open space or tank). As the driving module slowed down and stop when reached to Point B, it is proven that the programming is written correctly. 


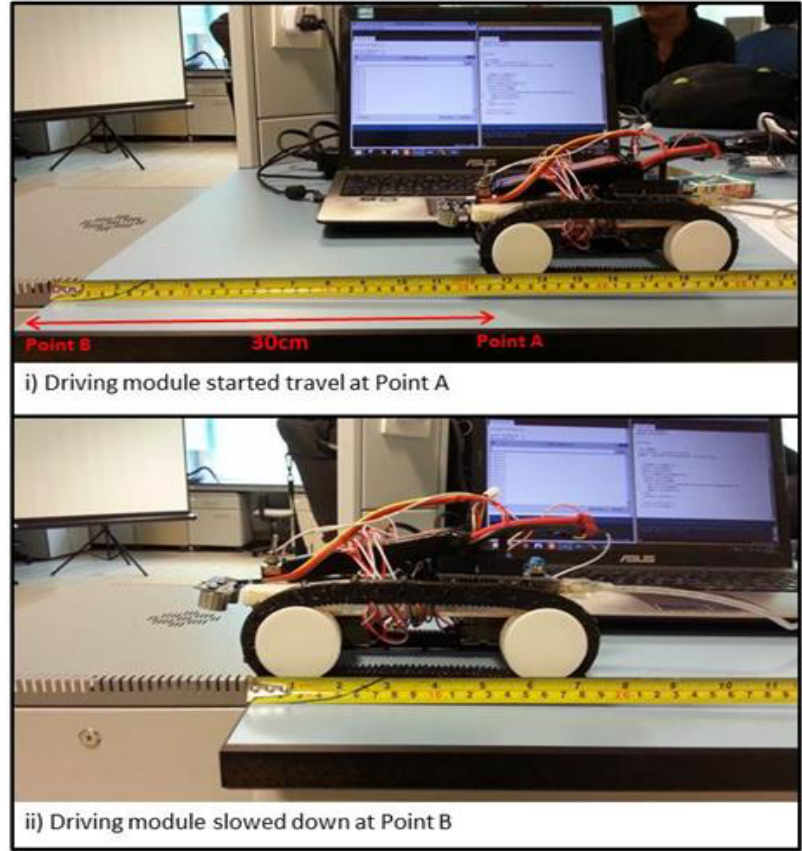

Figure 8 (i) and (ii) Experiment set up

Figures 9 and 10 show cornering movement of IPR-D300 during experiment. The figures are from the same experiment but different observed views. Objective of this experiment is to validate the hardware and software of the IPR-D300. Special modification has been made on the UPVC pipe elbow so that the movement of the robot in the pipe can be observed clearly. The IPR-D300 starts travelling with the same speed in the straight pipeline. When it reached to the corner, the driving wheel that located at the inner corner has slowed down. By changing the speed of each driving module according to its position in the elbow, the robot managed to make a transition and moving through the $90^{\circ}$ elbow pipeline. As shown in Figures 9 and 10, the robot successfully travel via $90^{\circ}$ elbow smoothly.

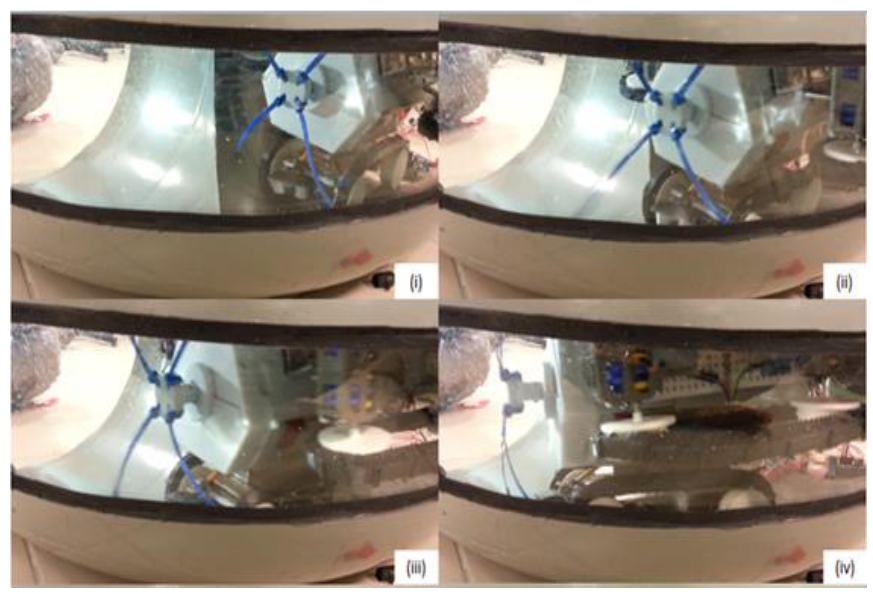

Figure 9 (i)-(iv) Test bed of $90^{\circ}$ elbow; side view

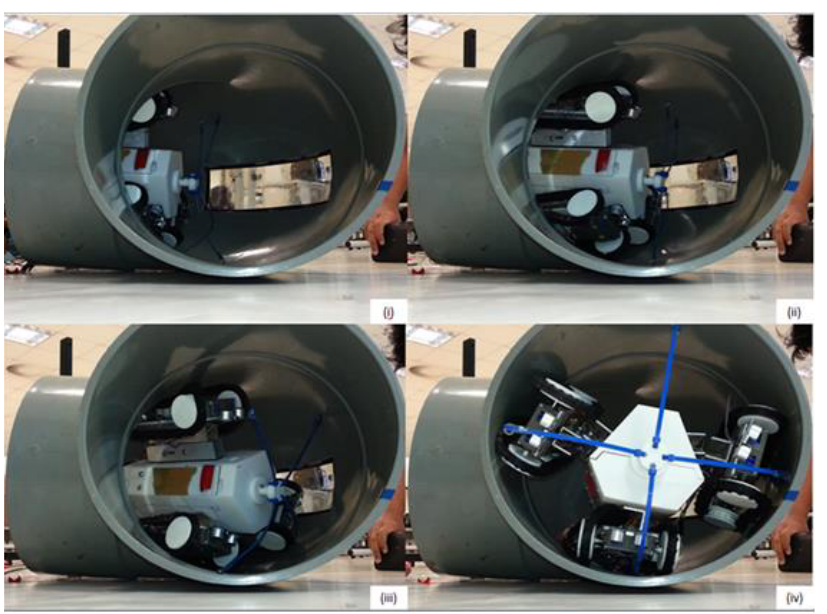

Figure 10 (i)-(iv) Test bed of $90^{\circ}$ elbow; front view

At the end of the experiment, one of the caterpillar wheel was out of the wheel track as shows in Figure 11. As the robot entering the elbow, the space at the sharp corner is decreasing and at the same time caterpillar wheel experienced additional pressure. Therefore, the additional pressure has squeezed, twisted and pushed the silicone wheel out of the wheel track. It is clearly shows that the silicone wheel was twisted and slipped from the wheel track.

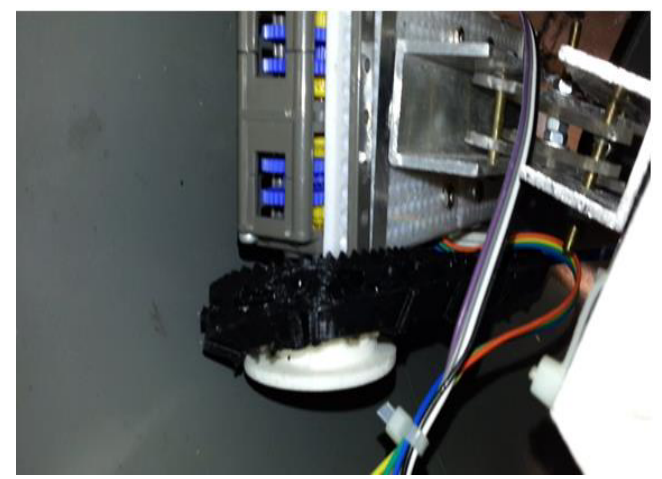

Figure 11 Deflection of silicon wheel

Another drawback of this prototype is unstable foldable linkage. Figures 12 and 13 show an imbalance linkage structure of the driving module. In order to ensure that the IPR-D300 is able to fit in $300 \mathrm{~mm}$ UPVC pipeline, the design has to be compact. Every part was measured accurately with the aim to minimize the robot size including structure of the linkage which is very minimum tolerance allowed. There is only 1 to $2 \mathrm{~mm}$ clearance at every joint that cause the link to shift slightly during compression. In the meantime, the foldable linkages have been fabricated with rough tolerance due to limited tools and equipment available in the workshop which is not suitable for tiny work piece. Furthermore, selection of $U$ shaped Aluminium is not suitable because it is big and heavy that consequently increase the overall weight structure and create an imbalance load distribution. Therefore, the U shaped Aluminium should be replaced 
with a lighter material. Realizing the potential of in-pipe robot for cleaning operation, the designated in-pipe robot has to meet the standard size of domestic pipeline which is $200 \mathrm{~mm}$. The size of the IPR-D300 has to be reduced to fit in the standard domestic pipeline.

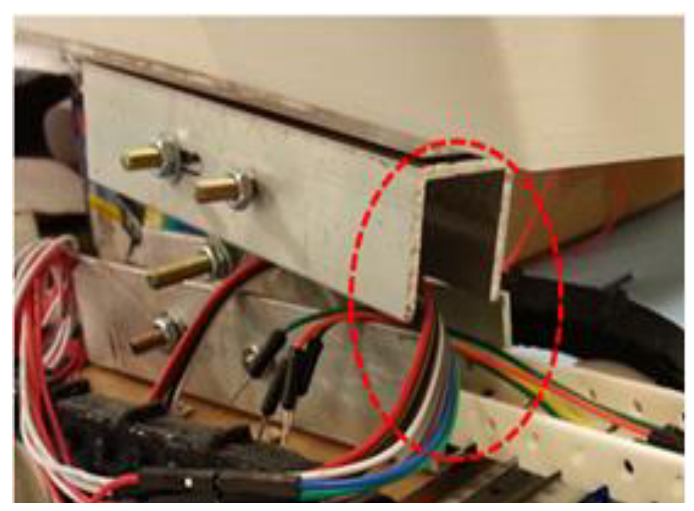

Figure 12 Imbalance foldable link, side view

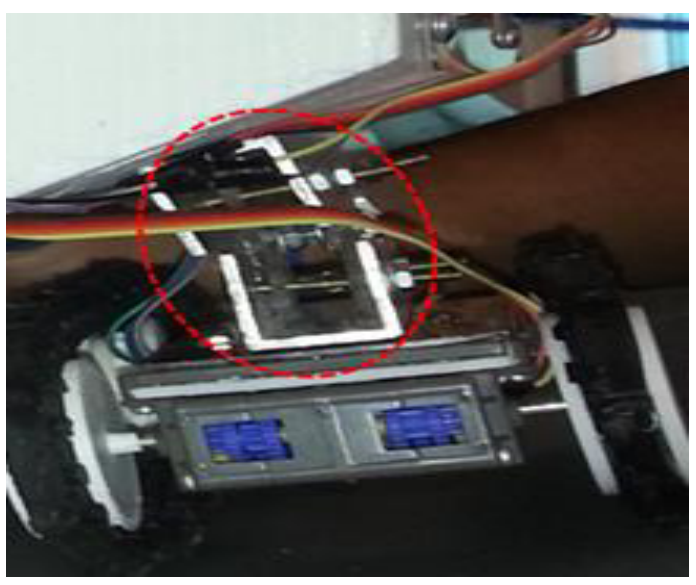

Figure 13 Imbalance foldable link; front view

\section{Conclusion}

As a conclusion, the characteristic of the $90^{\circ}$ pipeline cornering has been assessed and programmed. The hardware and software of the IPR-D300 has successfully developed and able to perform the cornering task. Likely, individual programmed motor through Adruino enable the IPR-D300 to vary its motor speed that consequently allow it to perform smooth cornering in $90^{\circ}$ pipe elbow. This paper is about the in-pipe robot for cornering mechanism that specifically discuss on how it is designed and programed. The concepts of controlling driving module independently facilitate the mechanism to overcome different gap radius as nature of the pipeline elbow, thus move smoothly. The mechanism is also designed to be integrated with other mechanism namely forward, backward and climbing to make it as complete in-pipe robot. Consequently, enable the robot to perform many other pipeline applications that required wireless in pipe robot for instance cleaning operation for centralized sewerage system.

\section{References}

1. Ismail I.N., Anuar A, Sahari K.S.M., Baharuddin M.Z., Fairuz M. \& Saad J.M.. Development of in pipe inspection robot: A review. In: Sustainable Utilization and Development in Engineering and Technology; October 2012; IEEE; p. 310-315. 2012.

2. Kakogawa A, and Ma S. Mobility of an In-pipe Robot with Screw Drive Mechanism Inside Curved Pipes. Robotics and Biometics (ROBIO; IEEE International Conference; 2010. p. 1530-1535); December 2010.

3. Nishimura T., Kakogawa A., \& Ma S. . Pathway selection mechanism of a screw drive in-pipe robot in T-branches. Automation Science and Engineering (CASE); August 2012; IEEE. p. 612-617; 2012.

4. Kakogawa A., \& Ma S. . Stiffness design of springs for a screw drive in-pipe robot to pass through curved pipes and vertical straight pipes. Advanced Robotics;26(3-4):253-276. 2012.

5. Daltorio, K. A., Gorb, S., Anrei, P., Andrew, D., Ritzmann, R. E. \& Quinn, R. D. . A Robot that Climbs Walls using Micro-structure ed Polymer Feet (M. O. Tokhi, G. S. Virk, \& M. A. Hossain, Eds.). Berlin, Heidelberg; Springer Berlin Heidelberg. 2006.

6. Kim, S., Spenko, M., Trujillo, S., Heyneman, B., Mattoli, V., \& Cutkosky, M. R. . Whole Body Adhesion: Hierarchical, directional and distributed control of adhesive forces for a climbing robot. Proceedings 2007 IEEE International Conference on Robotics and Automation. pp. 1268-1273. 2007.

7. Lee C. H., Joo D. M., Kim G. H., Kim B. S., Lee G. H., \& Lee S. G. . Elbow detection for localization of a mobile robot inside pipeline using laser pointers. In: Ubiquitous Robots and Ambient Intelligence (URAI), 2013 10th International Conference; IEEE; 2013. p. 71-75; October 2013.

8. Kim, J.H.. Design of a Fully Autonomous Mobile Pipeline Exploration Robot (FAMPER), Doctoral Dissertation, Department of Computer Science, Basic Science College, Faculty of Louisiana State University, Seoul National University of Technology. 2008.

9. Abidin A.S.Z., Aiman M.P.M.F, Muaz S.M., Hamizan Z.M., Sim C.C., Ashari M.F., Shahrol M., Annisa J., Muslimen R. and Syahmi J.M. Development of Track Wheel for In-pipe Robot Application. Journal Procedia Computer Science;76(2015):500-505. 2015.

10. Abidin A.S.Z., Hamizan Z.M., Aiman M.P.M.F, Muaz S.M., Sim C.C., Ashari M.F., Shahrol M., Annisa J., Muslimen R. and Syahmi J.M. and Chong Y.M. . Development of Cleaning Device for In-pipe Robot Application. Journal Procedia Computer Science. 2015;76(2015):506-511. 2015.

11. Roh S.G. and Choi H.R.. Differential Drive In-pipe Robot for Moving Inside Urban Gas Pipelines. Robotics, IEEE Transactions;21(1):1-17. 2005. 